\title{
A Simple Technique to Improve Microcrystals Using Gel Exclusion of Nucleation Inducing Elements
}

\author{
Adafih Blackburn ${ }^{1,2,+}$, Shahla H. Partowmah ${ }^{1,3,+}{ }^{1}$, Haley M. Brennan ${ }^{1,4,+}$, \\ Kimberly E. Mestizo ${ }^{1,5} \odot$, Cristina D. Stivala ${ }^{1,6} \oplus$, Julia Petreczky ${ }^{1,7}$, Aleida Perez ${ }^{1}$, \\ Amanda Horn ${ }^{1}$ D, Sean McSweeney ${ }^{8,9}$ and Alexei S. Soares ${ }^{8, *(D)}$ \\ 1 Office of Educational Programs, Brookhaven National Laboratory, Upton, NY 11973, USA; \\ ajblackburn98@gmail.com (A.B.); shahla.partow@gmail.com (S.H.P.); hmbrennan@email.wm.edu (H.M.B.); \\ Kimberly.mestizo@gmail.com (K.E.M.); cstivala19@ross.org (C.D.S.); julia.petreczky@gmail.com (J.P.); \\ pereza@bnl.gov (A.P.); ahorn@bnl.gov (A.H.) \\ 2 Department of Pharmacology, Stony Brook University, Stony Brook, NY 11794, USA \\ 3 School of Business, State University of New York at Old Westbury, Old Westbury, NY 11568, USA \\ 4 Department of Biology, College of William and Mary, Williamsburg, VA 23187, USA \\ 5 General Douglas MacArthur High School, Levittown, NY 11756, USA \\ 6 Ross School, East Hampton, NY 11937, USA \\ 7 Shoreham-Wading River High School, Shoreham-Wading River Central School District, Shoreham, \\ NY 11786, USA \\ 8 Photon Sciences Directorate, Brookhaven National Laboratory, Upton, NY 11973, USA; \\ smcsweeney@bnl.gov \\ 9 Biosciences Department, Brookhaven National Laboratory, Upton, NY 11973, USA \\ * Correspondence: soares@bnl.gov; Tel.: +1-(631)344-7306 \\ + These authors contributed equally to this work.
}

Received: 14 November 2018; Accepted: 10 December 2018; Published: 12 December 2018

check for updates

\begin{abstract}
A technique is described for generating large well diffracting crystals from conditions that yield microcrystals. Crystallization using this technique is both rapid (crystals appear in $<1 \mathrm{~h}$ ) and robust (48 out of 48 co-crystallized with a fragment library, compared with 26 out of 48 using conventional hanging drop). Agarose gel is used to exclude nucleation inducing elements from the remaining crystallization cocktail. The chemicals in the crystallization cocktail are partitioned into high concentration components (presumed to induce aggregation by reducing water activity) and low concentration nucleation agents (presumed to induce nucleation through direct interaction). The nucleation agents are then combined with $2 \%$ agarose gel and deposited on the crystallization shelf of a conventional vapor diffusion plate. The remaining components are mixed with the protein and placed in contact with the agarose drop. This technique yielded well diffracting crystals of lysozyme, cubic insulin, proteinase k, and ferritin (ferritin crystals diffracted to $1.43 \AA$ ). The crystals grew rapidly, reaching large size in less than one hour (maximum size was achieved in 1-12 h). This technique is not suitable for poorly expressing proteins because small protein volumes diffuse out of the agarose gel too quickly. However, it is a useful technique for situations where crystals must grow rapidly (such as educational applications and preparation of beamline test specimens) and in situations where crystals must grow robustly (such as co-crystallization with a fragment library).
\end{abstract}

Keywords: drug discovery; education; crystallization; crystallography; nucleation; micro-crystals; agarose; ferritin; lysozyme; proteinase k; insulin 


\section{Introduction}

It is convenient to have a robust crystallization protocol that yields high quality protein crystals even when the mother liquor is perturbed, for example by addition of chemicals from a fragment library [1,2]. Robust and rapid crystallization is also useful for applications such as to trouble-shooting diffraction equipment or in-classroom demonstrations. Here, we present a general technique for reliably and rapidly growing protein crystals by identifying elements that induce nucleation [3] and excluding these chemicals from the remaining crystallization solution by sequestering them in agarose gel [4]. This technique combines a vapor-diffusion approach to crystal growth [5] with a free-diffusion approach to nucleation [6]. A conventional vapor diffusion plate is used [7]. First, a pellet of gelled nucleation inducing chemicals is deposited on the crystallization shelf. Second, the solution of protein and precipitant is placed in contact with the pellet composed of gelled nucleation inducing chemicals. The reservoir contains the complete crystallization cocktail. If re-sealable crystallization plates are used (we used EasyXtal ${ }^{\circledR}$ 15-well crystallization plate from Qiagen), the gelled nucleation-inducing chemicals can be pre-deposited on the crystallization shelf so that crystal growth is initiated in one step by pipetting the stored solution of protein and precipitant into contact with the agarose. An added benefit is that hazardous nucleation-inducing chemicals are safer to handle when they are pre-applied as a gel to the crystallization shelf in a controlled laboratory environment (in contrast, precipitants frequently present few hazards if handled outside of the laboratory).

The protein experiences a concentration ratio for the gel sequestered nucleant that begins at 0 and terminates at 1 (where unity is the concentration in the reservoir). In contrast, the concentration of the precipitant varies by a smaller amount, from $\frac{1}{2}$ to 1 (again as a ratio of the concentration in the reservoir). A similar methodology was introduced when agar was first proposed as a crystallization facilitator [8], with the difference that all components were embedded in the agar matrix [9], and similar techniques continue to be used to simulate the advantages of growing crystals in microgravity [10]. In contrast, our goal is to introduce large concentration changes for nucleants, and more gradual concentration changes for precipitants. A comparable change in concentration can be induced in other ways, for example using button dialysis [11] and free diffusion methods [12]. The 4-corner method increases the likelihood of crystallization success by sampling four discreet concentrations of each precipitant [13]. One advantage of inducing large changes in concentration is that crystallization often becomes more robust. For example, co-crystallization in the presence of chemical libraries (such as fragment libraries) often perturbs conditions such that either no crystals are formed, or a shower of micro crystals are formed [14]. In contrast, inducing crystallization using a large precipitant gradient is often highly crystallogenic, and hence likely to robustly produce well diffracting crystals even when perturbed by chemical biology experiments. Robust conditions for co-crystallization are particularly valuable for drug discovery projects, where co-crystallization with chemicals of interest is often preferred over soaking because soaking can either take too much time [15] or can induce artificial artifacts [16].

\section{Materials and Methods}

We developed nucleant segregation strategies to crystallize four model proteins. Briefly, the crystallization cocktail is partitioned into two parts, and the nucleation component is segregated (using 2\% agarose) from the protein and remaining chemicals (Figure 1). Our first model protein was lysozyme, chosen because of the affordability and predictability that make it an unsurprising test sample in many development projects. For many of the same reasons our second model protein was proteinase K. Our third model protein was cubic insulin, to test the compatibility of nucleant segregation techniques with established protocols for button dialysis. Our final target for crystallization by exclusion of nucleating agents was ferritin. Because of its importance in general understanding of biological function, as well as its relevance to disease processes, ferritin is intensively studied by structural methods. However, ferritin is difficult to crystallize, which has slowed progress in structure-based understanding of its function and role in disease. Nucleant segregation offers a novel 
strategy for growing ferritin crystals. Our process induces the formation of small ferritin protein crystals in under one hour. Large and well diffracting ferritin crystals are obtained overnight. Using conventional techniques, comparable quality crystals require extended growth times (crystals may form more rapidly using techniques such as external magnetic fields; [17]).

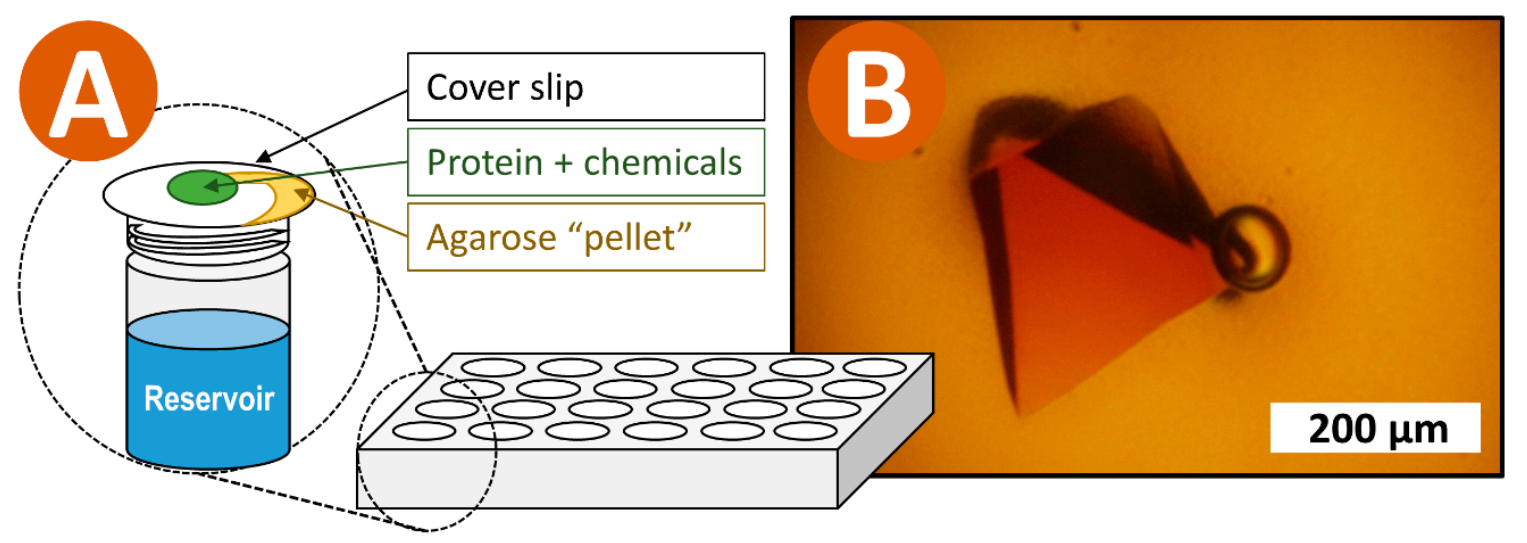

Figure 1. Crystallization using gel exclusion. (A) The gel exclusion setup that was used to crystallize test proteins (the shape of the agarose pellet shown was particularly important for ferritin). (B) Example of a ferritin crystal grown by gel exclusion (these crystals diffracted to $\sim 1.5 \AA$ resolution).

A faster crystallization protocol would be advantageous, for example for high throughput drug discovery projects (which require rapid crystal growth to screen large numbers of drug candidates). Fast and robust crystal growth can also be advantageous to basic science projects (for example, protein crystallization can be perturbed to generate insights into dynamic behavior).

\subsection{Lysozyme}

Agarose $(2 \% w / v)$ was heated to $100{ }^{\circ} \mathrm{C}$ and combined with $0.4 \mathrm{M}$ benzamidine hydrochloride,

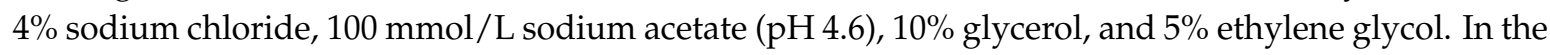
case of lysozyme, the entire crystallization cocktail was included in the nucleation pellet, as explained in Section 2.5. A $20 \mu \mathrm{L}$ pellet of gelled nucleation solution was deposited on each cover slip and allowed to cool (Figure 1A). Lysozyme powder (Sigma L6876, Sigma Aldrich, Saint Louis, MO, USA) was dissolved $(30 \mathrm{mg} / \mathrm{mL}$ in $100 \mathrm{mmol} / \mathrm{L}$ sodium acetate $\mathrm{pH} 4.6)$ and placed adjacent to the agarose pellet. Control crystals were grown in a similar way, with the difference that no agarose was added, such that the nucleating solution was added in the liquid state.

\subsection{Proteinase $K$}

Agarose $(2 \% w / v)$ at $100{ }^{\circ} \mathrm{C}$ was combined with $0.8 \mathrm{M}$ sodium nitrate. A $20 \mu \mathrm{L}$ pellet of this gel was deposited on each cover slip and allowed to cool. Proteinase K powder (Sigma P2308) was dissolved $\left(30 \mathrm{mg} / \mathrm{mL} 0.1 \mathrm{M}\right.$ BisTris $\mathrm{pH} 6.5$ and $0.08 \mathrm{M} \mathrm{CaCl}_{2}$ ) and placed adjacent to the agarose pellet. Similar to the growth of Lysozyme control crystals, proteinase K control crystals were grown with no agarose and the addition of nucleating solution was in the liquid state.

\subsection{Cubic Insulin}

Gelled nucleating solution $(2 \% w / v$ agarose $)$ at $100{ }^{\circ} \mathrm{C}$ was combined with $0.25 \mathrm{~mol} / \mathrm{L}$ sodium phosphate $\mathrm{pH} 9.2$ and deposited on individual cover slips as $20 \mu \mathrm{L}$ pellets to cool. Human insulin powder (Sigma 91077C) was dissolved (30 mg/mL in $0.05 \mathrm{~mol} / \mathrm{L}$ sodium phosphate $\mathrm{pH} 11)$ and placed adjacent to the pellet. Control crystals for insulin were not grown using previous techniques. Button dialysis [18] proved more effective with the button containing $20 \mu \mathrm{L}$ of $30 \mathrm{mg} / \mathrm{mL}$ protein in $0.05 \mathrm{~mol} / \mathrm{L}$ sodium phosphate $\mathrm{pH} \mathrm{11}$, and the reservoir (around the button) containing excess of $0.25 \mathrm{~mol} / \mathrm{L}$ sodium phosphate $\mathrm{pH}$ 9.2. 


\subsection{Ferritin}

Mammalian ferritin assembles into a roughly spherical protein that is primarily responsible for oxidizing soluble ferrous iron into insoluble ferric iron $\left(\mathrm{Fe}^{2+} \rightarrow \mathrm{Fe}^{3+}\right.$ is the ferroxidase activity). Ferritin then stores the insoluble iron in a $270 \mathrm{~nm}^{3}$ cavity that can accommodate up to 4500 iron atoms [19]. Ferritin consists of 24 heterogeneous subunits, each of which can be either a light subunit (FTL ferritin light chain) or a heavy subunit (FTH1 - ferritin heavy chain) [20]. FTL and FTH1 subunits are structurally similar, but FTH1 has increased ferroxidase activity, so that the greater the proportion of FTH1, the greater the ability of the ferritin assembly to rapidly incorporate iron [21]. Ferritin is intensively studied for two primary health related reasons. First, ferritin accumulates iron in response to infection (denying iron to invaders) [22]. Second, ferritin maintains a constant level of soluble iron (iron homeostasis), which is important in anemia and iron overload defects [23]. In addition to its direct applications to human health, ferritin's bio-mineralization and metal accumulation properties are frequently used as tools in biotechnology applications [24].

Cadmium is required for formation of ferritin crystals. In conventional crystallization, the cadmium is introduced along with the remaining crystallization chemicals. Since cadmium is very effective in inducing nucleation of ferritin crystals, this process requires a very fine balance for the cadmium concentration. If there is too much cadmium, a shower of microcrystals results. If there is too little, the protein either remains in solution, or grows exceedingly slowly. One consequence of this, is that for most protocols, much of the protein remains un-crystallized, since the crystallization drop runs out of cadmium much before it runs out of ferritin protein.

We first used conventional high throughput screening to identify crystallization conditions for ferritin (cadmium in different concentrations was included in all of our screens). We used established micro-crystallization protocols [25] using an acoustic screening robot [26] to identify three conditions that yielded ferritin crystals. We then used gel exclusion to segregate the cadmium from the remaining crystallization cocktail to identify the optimal crystallization condition for our method (3.5 mol/L $\left(\mathrm{NH}_{4}\right)_{2} \mathrm{SO}_{4}, 100 \mathrm{mmol} / \mathrm{L}$ tris(hydroxymethyl)aminomethane (TRIS) $\mathrm{pH} 7.4,1 \mathrm{mmol} / \mathrm{L}$ ethylenediaminetetraacetic acid (EDTA), $50 \mathrm{mmol} / \mathrm{L} \mathrm{CdSO}_{4}$. The cadmium was sequestered in a hydrogel by combining with $2 \%$ agarose and heating to $100{ }^{\circ} \mathrm{C}$ for $5 \mathrm{~min}$.

We initially used a conventional "hanging drop" crystallization strategy, which consisted of a plate with 24 separate crystallization chambers. The protein + crystallization chemicals + cadmium-agarose pellet were positioned on the cover-slip. The cadmium-agarose pellet was of approximately equal size compared to the protein-chemical drop, and was placed on the cover-slips and left to equilibrate with the reservoir. The protein-chemical drop consisted of 50\% ferritin and $50 \%$ crystallization solution. Once we identified an optimal crystallization condition, we began experimenting with altering the shape of the agar pellet, to further optimize crystallization. We used screw-on cover slips (because conventional cover slips contain limited real estate for designing different agar pellet shapes (screw-on cover slips also have the advantage of a plastic boundary that can be used in the shaping process; EasyXtal ${ }^{\circledR} 15$-Well from Qiagen). We observed that an extended shape, which minimized the contact area between the cadmium-pellet and the crystallization solution, was optimal (Figure 1B). For comparison, control crystals were grown without using agarose.

\subsection{Lysozyme Co-Crystallization with Fragment Library}

Each chemical from a 48-fragment library was deposited on a cover slip and allowed to dry (10 $\mu \mathrm{L}$ of $100 \mathrm{mmol} / \mathrm{L}$ fragment dissolved in water). Gelled nucleating solution ( $2 \% w / v$ agarose) was heated to $100{ }^{\circ} \mathrm{C}$ and combined with $0.4 \mathrm{~mol} / \mathrm{L}$ benzamidine hydrochloride, $4 \%$ sodium chloride, $100 \mathrm{mmol} / \mathrm{L}$ sodium acetate ( $\mathrm{pH} 4.6), 10 \%$ glycerol, and 5\% ethylene glycol. Then, $20 \mu \mathrm{L}$ pellets of the gel was deposited on each cover slip and allowed to cool while in contact with each chemical. Lysozyme powder (Sigma L6876) was dissolved $(30 \mathrm{mg} / \mathrm{mL}$ in $100 \mathrm{mmol} / \mathrm{L}$ sodium acetate $\mathrm{pH} 4.6$ ) and placed adjacent to the agarose pellet. Control crystals were grown with no agarose added, having nucleating solution added in the liquid state. 
The cover slips containing lysozyme, proteinase, and cubic insulin were placed on a VDX ${ }^{\mathrm{TM}}$ hanging drop plate (Hampton HR3-172, Hampton Research, Aliso Viejo, CA, USA), where the reservoir contained the complete respective crystallization cocktail. Crystallization plates and buttons were placed on a Leica MZ16 microscope (Leica Camera AG, Wizlar, Germany) equipped with an Olympus DP72 camera (Olympus, Shinjuku, Tokyo, Japan) operated with the cellSens standard (version 1.6, Olympus, Shinjuku, Tokyo, Japan) software. Pictures of the growing crystals were taken automatically using the process manager at intervals of $150 \mathrm{~s}$ (gel exclusion crystals) and $1500 \mathrm{~s}$ (control crystals). Growth rate data were obtained for three gel exclusion crystals and for three control crystals of each protein, and an overall crystal length " $\mathrm{L}$ " was determined for each crystal at each time point as described in Section 2.2. Cubic insulin crystals moved through the field of view during the growth process, so a single continuous growth curve could not be produced. Three student researchers measured the dimensions of each crystal by inspection using Image J software (version 1.51w, NIH, Bethesda, MD, USA) (the measurements from the three students were then averaged). The crystal volume was determined using the average $x, y$, and $z$ dimensions of each crystal, and the cube root of the crystal volume was used as a measure of the observed length " $\mathrm{L}$ " of the crystal at each time point (Equation (1), Section 3).

\section{Results}

We observed that all crystals that we tested grew faster using gel exclusion compared to controls grown using conventional techniques. For both gel exclusion and controls, the crystal size was initially observed to increase according to a one term asymptotic function but was bounded by an upper limit that is less than the asymptotic value. Hence, the observed crystal size was empirically fitted to a two-part equation, where the initial growth follows a simple two-parameter asymptotic Function (1a) until reaching a maximum size limit, after which crystal size is constant (1b):

$$
\left[\begin{array}{c}
L=L_{c}\left(\frac{t}{t+\tau}\right) \text { if } L<L_{\max } \\
L=L_{\max } \text { if } L>L_{\max }(1 a) \\
(1 b)
\end{array}\right]
$$

Here $L$ is the predicted length of each side of the crystal, $L_{\mathrm{c}}$ is an adjustable constant that corresponds to the asymptotic value (length units), $t$ is the time over which crystallization has occurred, $\tau$ is an adjustable constant that corresponds to the time needed for the crystal to reach $\frac{1}{2} L_{\mathrm{c}}$ (time units), and $L_{\max }$ is an adjustable constant which indicates the upper limit for crystal size (length units, the point at which all soluble protein has been subsumed into the crystal such that no further growth is possible). The three adjustable constants were iteratively refined to minimize the discrepancy between the observed crystal length and the predicted crystal length (Table 1).

Compared to controls, lysozyme crystals grown using gel exclusion grew 4.6 times faster. Also, when combined with a 48 -fragment library, $100 \%$ of the gel exclusion lysozyme co-crystals grew, compared with $38 \%$ of the control lysozyme crystals. Proteinase K crystals were also observed to grow faster using gel exclusion, compared to controls (10.7 times faster).

Cubic insulin is conventionally grown using button dialysis, which involves a laborious setup of the apparatus. In contrast, our gel exclusion crystallization setup was simple, and resulted in faster growing crystals (17.1 times faster).

Growing ferritin crystals requires a prolonged period of time to grow using conventional methods, particularly for iron containing ferritin. Using gel exclusion, we were able to grow large well diffracting ferritin crystals overnight. These crystals diffracted to higher resolution than any iron containing ferritin crystal in the protein data bank (PDB), $1.43 \AA$, compared to $2.22 \AA$; our best control crystal had a resolution of $2.54 \AA$. The highest resolution for iron containing equine ferritin in the PDB is $2.2 \AA$, which is comparable to our best control crystals at $2.5 \AA$. Data to $2.0 \AA$ have been recorded from iron containing ferritin crystals grown under high magnetic fields but were not deposited in the PDB [27]. 
Table 1. Refined curve fitting parameters for crystal growth curves.

\begin{tabular}{ccc}
\hline Lysozyme: & Gel Exclusion & Hanging Drop \\
\hline Time step $(\mathrm{s})$ & 150 & 1500 \\
Tau $(\mathrm{h})$ & $0.57 \pm 0.02$ & $2.66 \pm 0.48$ \\
Lmax $(\mu \mathrm{m})$ & $512 \pm 49$ & $427 \pm 71$ \\
Lc $(\mu \mathrm{m})$ & $605 \pm 58$ & $505 \pm 84$ \\
Residual $(\%)$ & $2.04 \% \pm 0.36 \%$ & $2.55 \% \pm 0.56 \%$ \\
\hline Proteinase K: & Gel Exclusion & Hanging Drop \\
\hline Time step $(\mathrm{s})$ & 120 & 600 \\
Tau $(\mathrm{h})$ & $1.01 \pm 0.24$ & $16.16 \pm 2.60$ \\
Lmax $(\mu \mathrm{m})$ & $363 \pm 81$ & $361 \pm 31$ \\
Lc $(\mu \mathrm{m})$ & $430 \pm 21$ & $534 \pm 46$ \\
Residual $(\%)$ & $5.66 \% \pm 0.82 \%$ & $2.80 \% \pm 0.06 \%$ \\
\hline Cubic insulin: & Gel Exclusion & Hanging Drop \\
\hline Time step $(\mathrm{s})$ & 150 & 1500 \\
Tau $(\mathrm{h})$ & $3.30 \pm 0.00$ & $56.33 \pm 5.18$ \\
Lmax $(\mu \mathrm{m})$ & $222 \pm 16$ & $86 \pm 10$ \\
Lc $(\mu \mathrm{m})$ & $555 \pm 40$ & $214 \pm 25$ \\
Residual $(\%)$ & $3.14 \% \pm 0.63 \%$ & $4.92 \% \pm 1.97 \%$ \\
\hline
\end{tabular}

\subsection{Lysozyme}

Lysozyme crystals grew much faster using gel exclusion compared to controls. Crystals' growth using gel exclusion and controls had similar crystal habits, though the gel exclusion crystals tended to exhibit a less elongated crystal habit (Figure 2). The growth constants $\tau, L_{\mathcal{C}}$, and $L_{\max }$ (Equation (1)) for each of the three crystals grown using gel exclusion, and for each of the three crystals grown using conventional methods, are shown in Table 1. For visual illustration, we also fitted growth curves to the overall data obtained by averaging the lengths of all three crystals grown using gel exclusion and compared to the overall growth curve from the control crystals (Figure 1 inset).

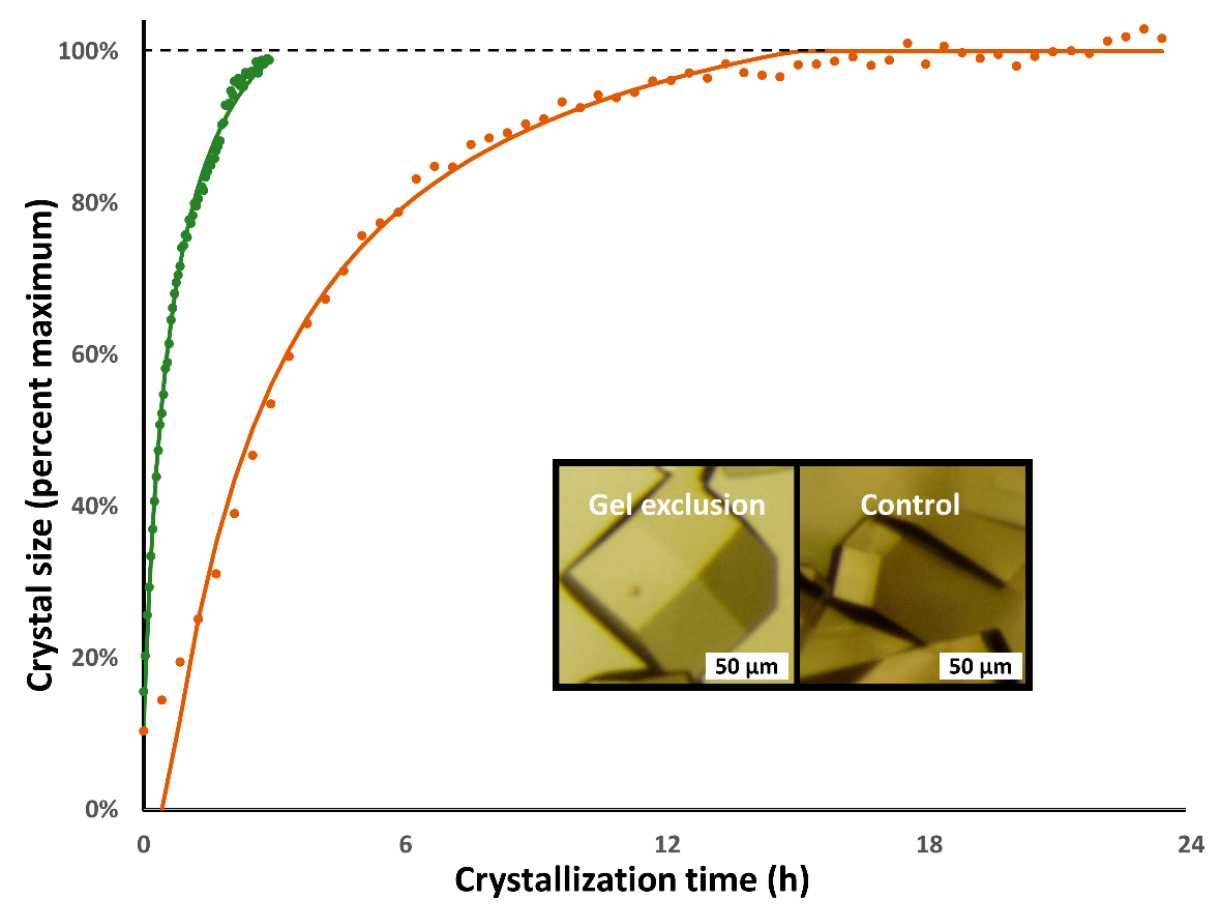

Figure 2. Growth rate for lysozyme crystals grown using gel exclusion (green) and conventional hanging drop (orange). Data are fitted by a single polynomial asymptotic curve with an upper bound that is lower than the asymptotic value. Crystal habits were similar (inset). 


\subsection{Proteinase $K$}

Proteinase $\mathrm{K}$ crystals also grew much faster using gel exclusion compared to controls. Crystal growth using gel exclusion and controls led to virtually identical crystal habits (Figure 3), and the fitted parameters are shown in Table 1. As in Section 2.1, overall growth rates were also determined by averaging the sizes of the three crystals grown using gel exclusion and the three controls (Figure 3 inset).

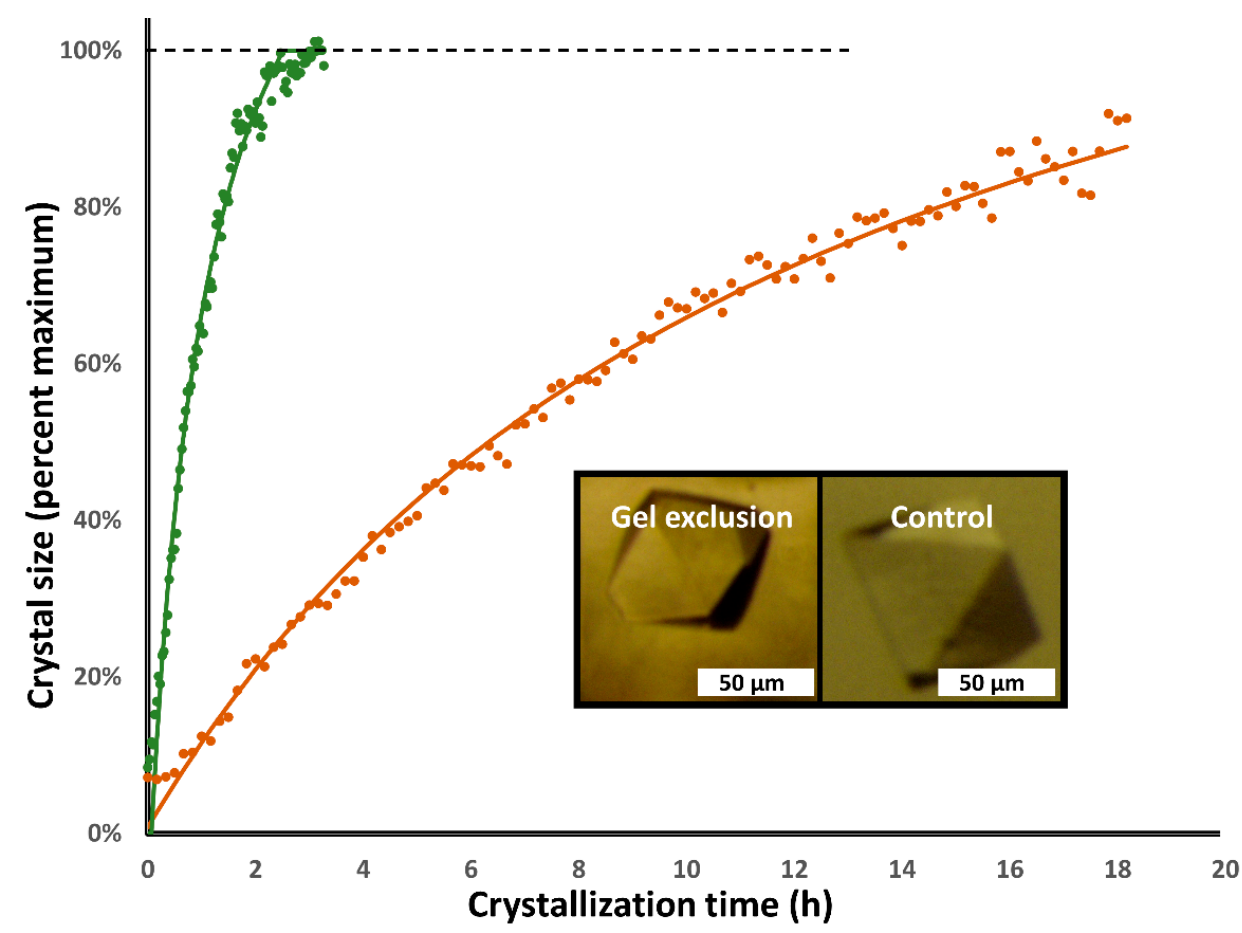

Figure 3. Growth rate for proteinase K crystals grown using gel exclusion (green) and conventional hanging drop (orange). Data are fitted by a single polynomial asymptotic curve with an upper bound that is lower than the asymptotic value. Crystal habits were virtually identical (inset).

\subsection{Cubic Insulin}

Gel exclusion proved to be a simple alternative to button dialysis, with many of the same benefits, but with a much simpler experimental setup. Cubic insulin crystals grown using both methods had identical habits. As previously, crystal growth was greatly accelerated using gel exclusion (average values in Table 1; continuous growth curves could not be generated due to crystal movement).

\subsection{Ferritin}

Using gel exclusion to sequester cadmium to fine-tune crystallization of ferritin yielded well diffracting crystals that grew in a few hours. As mentioned in Section 2.4, we noticed that there was a correlation between the shape of the cadmium pellet and the quality of the crystals that grew in that crystallization drop. After exploring many different shapes for the cadmium pellet, we observed that the optimum shape was a "comma" shape. The crystals that resulted from this improved procedure had a better, more symmetric crystal habit, and yielded improved diffraction quality.

Once we chose an optimal crystallization strategy (Section 2.4), we obtained an X-ray diffraction data set (Figure 4). Diffraction data were measured at the National Synchrotron Light Source II (NSLS II) beamline 17-ID-1 (AMX) and processed with XDS (version January 2018, MPI, Heidelberg, Germany) [28]. Data were further processed using CTRUNCATE in the CCP4i suite (version 0.8.9, CCP4, Oxon, UK) [29]. Structures were obtained by molecular substitution from published models and refined using REFMAC (v0.8.9, CCP4, Oxon, UK) [30] and ArpWarp [31] (version 7, EMBL, Hamburg, Germany) (starting models 1IER [32]). Structures were visually inspected using coot (version 0.8.9, 
CCP4, Oxon, UK) [33]. The quality of these crystals was a significant improvement compared to the best crystals grown using conventional crystallization strategies (Table 2; PDB accession code 6MSX). We conclude that our proposed three-state crystallization strategy (with three distinct chemical environments in the hanging drop, the agar pellet, and the reservoir) allowed us to fine-tune the crystallogenesis of ferritin, yielding faster growing and higher quality crystals.

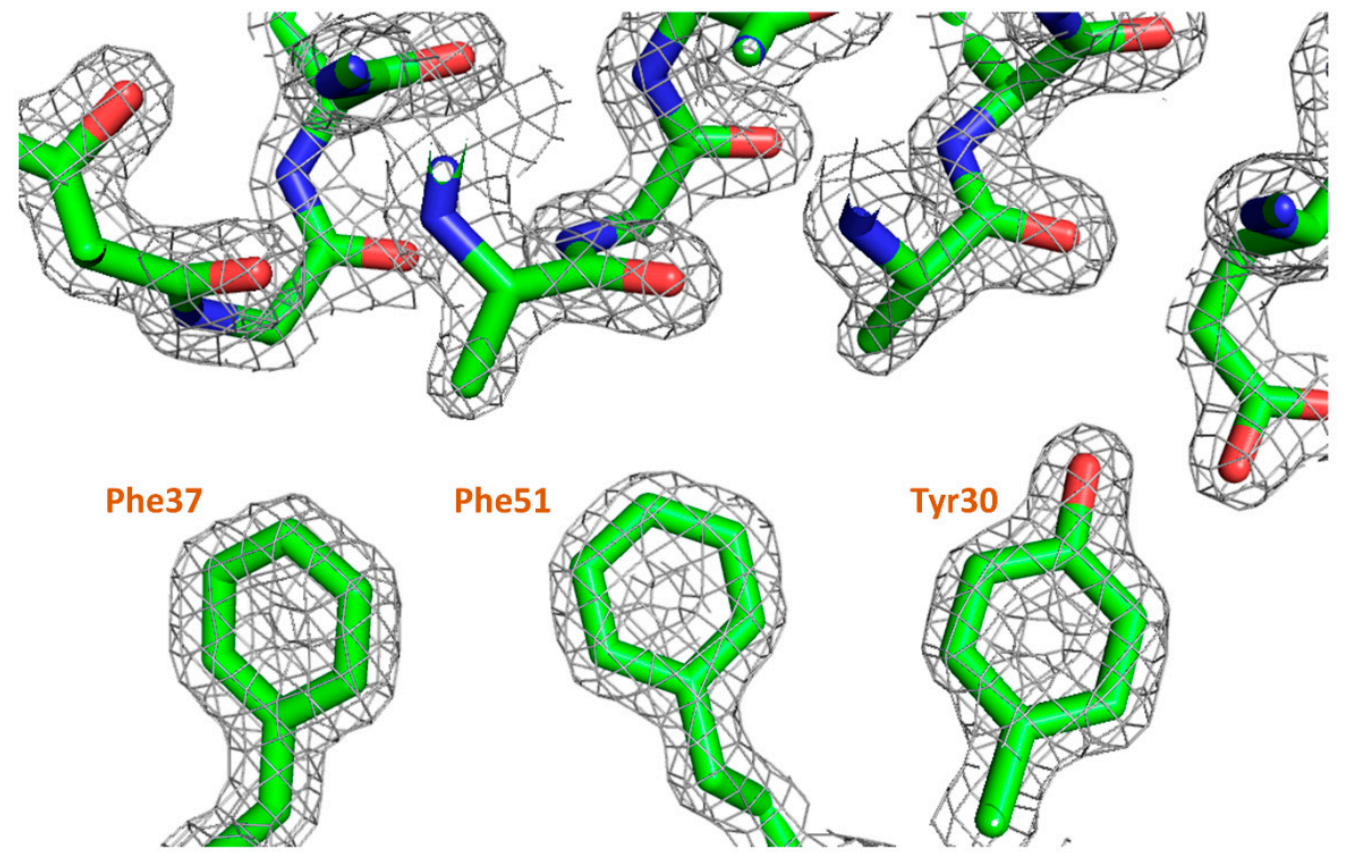

Figure 4. High quality crystals grown in $12 \mathrm{~h}$ yield accurate electron density for ferritin. Electron density is contoured at $3 \sigma(2 \mathrm{fo}-\mathrm{fc})$. Three residues are shown, the remainder of the ferritin is represented as a helix ribbon (6MSX).

Table 2. Data reduction and structure refinement statistics.

\begin{tabular}{ccccc}
\hline Protein & Ferritin & Lysozyme & Lysozyme & Lysozyme \\
\hline Fragment & $\mathrm{n} / \mathrm{a}$ & $\begin{array}{c}\text { 3-amino- } \\
\text { Phenol }\end{array}$ & $\begin{array}{c}\text { Picolinic } \\
\text { acid }\end{array}$ & $\begin{array}{c}\text { Eosin } \mathrm{Y} / \\
\text { Trp62 }\end{array}$ \\
Resolution $(\AA)$ & $1.43(1.47)$ & $1.35(1.39)$ & $1.23(1.26)$ & $1.56(1.60)$ \\
$\mathrm{R}_{\text {merge }}(\%)$ & $11.6(407)$ & $7.0(74.7)$ & $6.7(53.0)$ & $9.0(69.4)$ \\
$\mathrm{CC}_{1 / 2}$ & $100.0(49.9)$ & $99.5(84.6)$ & $99.4(82.4)$ & $99.5(77.7)$ \\
$<\mathrm{I} / \sigma(\mathrm{I})>$ & $31.81(1.17)$ & $21.9(3.2)$ & $17.4(2.3)$ & $17.8(3.1)$ \\
Multiplicity & $138.2(48.9)$ & $12.8(11.3)$ & $8.1(5.5)$ & $12.8(11.2)$ \\
Unique reflections & 47,743 & $27,063(1906)$ & $32,856(1997)$ & $17,660(1235)$ \\
Completeness $(\%)$ & $100.0(100.0)$ & $99.7(96.0)$ & $97.3(83.1)$ & $99.7(96.3)$ \\
Rwork $(\%)$ & 15.71 & 15.75 & 17.62 & 17.44 \\
Rfree $(\%)$ & 17.17 & 18.59 & 19.91 & 21.16 \\
R.M.S. bond $(\AA)$ & 0.03 & 0.03 & 0.03 & 0.02 \\
rms angles $\left({ }^{\circ}\right)$ & 2.65 & 2.53 & 2.51 & 1.83 \\
Mean B $\left(\AA^{2}\right)$ & 30 & 19 & 19 & 26 \\
Mean ligand B $\left(\AA^{2}\right)$ & $\mathrm{n} / \mathrm{a}$ & 17 & 30 & 38 \\
Figure & 4 & $6 \mathrm{a}$ & $6 \mathrm{~b}$ & $6 \mathrm{c}$ \\
PDB code & $6 \mathrm{MSX}$ & $6 \mathrm{MX} 9$ & $\mathrm{n} / \mathrm{a}$ & $\mathrm{n} / \mathrm{a}$ \\
\hline
\end{tabular}

\subsection{Lysozyme Co-Crystallization with Fragment Library}

We originally designed the gel exclusion method as a convenience to easily generate crystals for applications that require rapid crystal growth, such as educational courses and benchmarking the performance of diffraction instrumentation. However, during testing we observed that gel exclusion 
was a robust crystallization technique that reliably yielded crystals even when mistakes were made during the crystallization setup. To test the usefulness of this observation, we co-crystallized lysozyme with 48 chemical fragments using gel exclusion and compared this to crystals generated using conventional hanging drop. Using gel exclusion, we observed lysozyme crystals in all 48 fragment screens (Figure 5, top), compared to 26 in the 48-control screen (Figure 5, bottom).

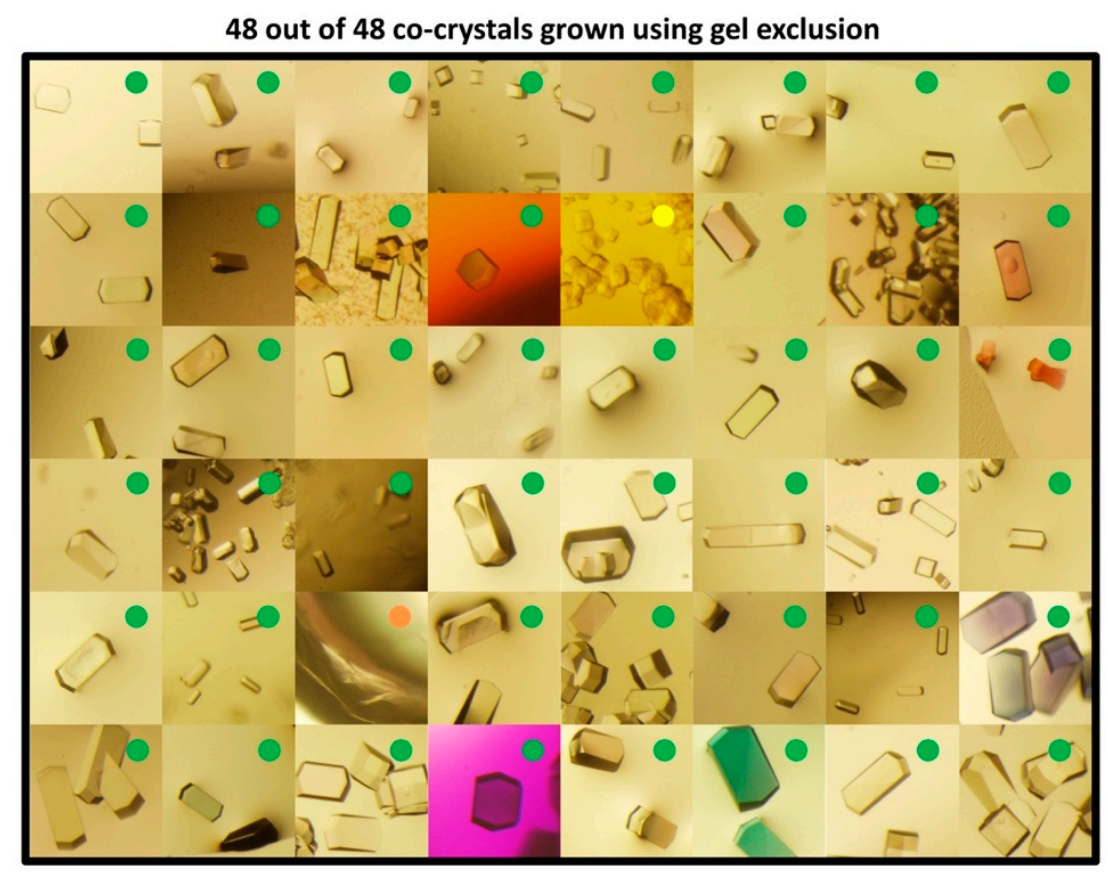

26 out of 48 co-crystals grown using hanging drop

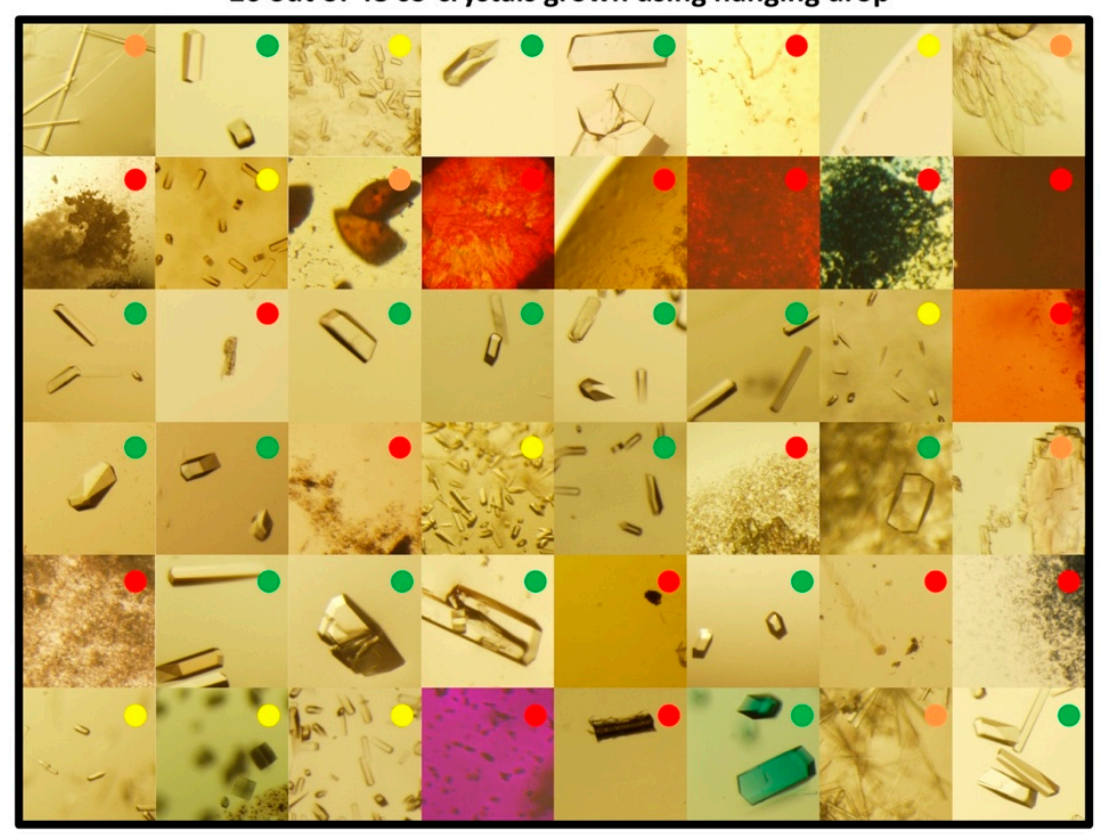

Figure 5. Co-crystallization of lysozyme with 48 fragments (gel exclusion on left, control on right). Green dots indicate large crystals, yellow dots indicate small crystals, orange dots indicate possible crystals, and red dots indicate no crystals). Gel exclusion co-crystallization yielded 48 crystals out of 48 screens, compared to 26 using conventional hanging drop (detailed description of fragment library in Supplementary materials section 1). 
We obtained diffraction data from crystals drawn from all 48 gel exclusion screens. Diffraction data were measured and processed, and structures were solved, as described in Section 2.5 (starting models 4N8Z; [34]). Inspection of the structures revealed that three of the fragments tested induced observable changes in the electron density, 3-aminophenol, picolinic acid, and eosin Y (Figure 6A-C respectively). One observed ligand (3-aminophenol) was deposited in the PDB (6MX9).

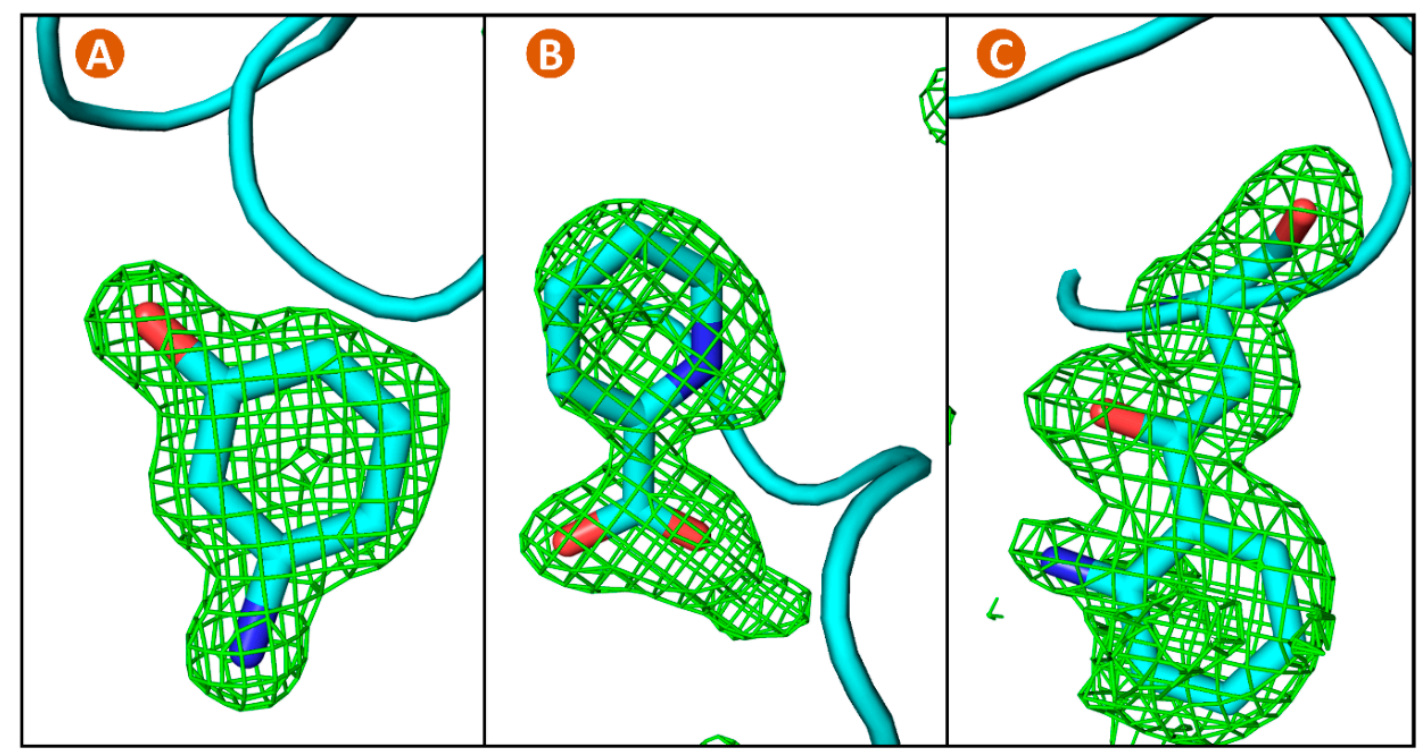

Figure 6. Electron density indicated that 3 out of 48 fragments perturbed the lysozyme starting model. Two of these were observed bound to the protein, (A) 3-aminophenol and (B) picolinic acid. (C) The third induced oxidation of tryptophan 62 (eosin Y) (C). Figure (C) depicts a tryptophan residue with an open ring and a changed confirmation (observed in the presence of eosin $\mathrm{Y}$ ) [35]. Omit difference electron density is contoured at $3 \sigma(\mathbf{A})$ or $2 \sigma(\mathbf{B}, \mathbf{C})$. Since picolinic acid resembles a crystallization component, (B) was re-crystallized without benzamidine.

\section{Discussion}

As discussed earlier, agar exclusion is a useful strategy for applications that require fast crystallization, such as educational applications and generating test crystals to test diffraction equipment. There are also safety advantages to sequestering toxic chemicals inside of an agar pellet (such as cadmium sulfate used to nucleate ferritin crystals), and further advantages in work flow compared to awkward crystallization setups such as button diffusion. In the case of ferritin, crystals not only grew very quickly, but yielded higher resolution data than conventionally grown crystals. High resolution data can improve the accuracy of ferritin models deduced from the diffraction (for example, we used the diffraction data to estimate the iron content inside the ferritin cavity using an iterative density modification and phase refinement procedure that is sensitive to data and model quality [36]). The integrated total number of iron atoms inside of the ferritin cavity was deduced to be $835 \pm 22$, starting from 20 models with very different initial average cavity density. This number is similar to a previously reported estimate of $800 \mathrm{Fe}$ /molecule for native equine ferritin (measured using atomic absorption spectrophotometry $[37,38])$. All of these advantages are incremental conveniences that improve experimental timing, work flow, or quality—but do not potentiate new science that would otherwise not be possible.

The 100\% co-crystallization rate for lysozyme with 48 fragments suggests that agar exclusion may be a robust approach to fragment screening. The fact that none of the three fragments that induced observable perturbations to the protein structure yielded crystals in the conventional hanging drop screens begs the question of whether fragments that interact with the protein surface are more likely to interfere in the crystallization process, compared to fragments that do not interact with the protein surface. This would be particularly important because it would imply that the miss rate for fragment 
screening is exaggerated because useful fragments are especially likely to interfere in co-crystallization. We can use the hypergeometric distribution to calculate that there is a $9 \%$ probability of observing 0 perturbations in the electron density from the set of 26 co-crystals that did grow in the control set, and observing 3 perturbations in the electron density from the set of 22 co-crystals that did not grow in the control set, if failure to co-crystallize and perturbation of the electron density are independent variables (see supplemental section 2). Hence, we conclude that lysozyme co-crystals are less likely to form in the presence of chemicals that perturb the structure of lysozyme, compared to chemicals that do not perturb the structure of lysozyme.

\section{Conclusions}

We found simple and effective partitions of the crystallization cocktail for four test proteins, with some of the chemicals sequestered in an agar pellet and some present in the protein drop. We observed that this gel exclusion approach yielded large well diffracting crystals far more rapidly than conventional crystallization. In the case of ferritin, crystals grown using gel exclusion were of higher quality than comparable crystals grown using hanging drop, and also of higher quality than iron containing crystals in the protein data bank. In the case of lysozyme, we observed that co-crystallization with a fragment library was more robust with agar exclusion compared to conventional hanging drop techniques. Notably, none of the three fragments that were observed to perturb the lysozyme structure resulted in crystals when co-crystallized using conventional hanging drop methods. We conclude that, in the case of proteins that are available in high quantities, agar exclusion is a promising technique for rapidly growing robust protein crystals.

Supplementary Materials: The following are available online at http:/ / www.mdpi.com/2073-4352/8/12/464/s1.

Author Contributions: Conceptualization, A.S.S.; investigation, A.S.S., A.B., S.H.P., H.M.B., K.E.M., C.D.S. and J.P.; writing-original draft preparation, A.S.S.; writing-review and editing, A.S.S., A.B., S.H.P., H.M.B., K.E.M., C.D.S., J.P., A.P., A.H. and S.M. supervision, A.S.S., S.H.P., A.P., A.H. and S.M.

Funding: Major ongoing financial support for acoustic droplet ejection applications was through the Life Science Biomedical Technology Research resource, supported by the National Institute of Health, National Institute of General Medical Sciences (NIGMS) through a Biomedical Technology Research Resource P41 grant (P41GM111244), and by the DOE Office of Biological and Environmental Research (KP1605010).

Acknowledgments: Data for this study were measured at beamline 17-ID-1 (AMX) at the National Synchrotron Light Source II (NSLS II). Personnel for this study were recruited largely through the Science Undergraduate Laboratory Internships Program (SULI) and the High School Research Program (HSRP), supported through the U.S. Department of Energy, Office of Science, Office of Workforce Development for Teachers and Scientists (WDTS), DOE contract No. DE-SC0012704. SULI students contributed during the 2017 summer + fall, and during the 2018 summer. HSRP students contributed during the 2018 summer. Initial high throughput screening was performed during the 2017 Summer Science Exploration "Structural Biology: Solving Protein Structures at the Synchrotron" (supported by A. Horn). We thank the participants B. Scapellati, A. Morrison, R. Barnes, A. DeLury, S. Smiley, H. Dutt, J. Hart, J. Caesar, A. McCrea, R. Tiska, M. De Somma, M. De Somma, M. McKenna, S. Wallach, L. Davis, A. Dolich, T. Giannuzzi, R. Nagia, and Alyssa Arbuiso.

Conflicts of Interest: The authors declare no conflict of interest.

\section{References}

1. Lin, Y. What's happened over the last five years with high-throughput protein crystallization screening? Expert Opin. Drug Discov. 2018, 13, 691-695. [CrossRef]

2. Chayen, N.E.; Helliwell, J.R.; Snell, E.H. Macromolecular Crystallization and Crystal Perfection; Oxford University Press: Oxford, UK, 2010; Volume 24.

3. Santesson, S.; Cedergren-Zeppezauer, E.S.; Johansson, T.; Laurell, T.; Nilsson, J.; Nilsson, S. Screening of nucleation conditions using levitated drops for protein crystallization. Anal. Chem. 2003, 75, 1733-1740. [CrossRef] [PubMed]

4. Ericson, D.L.; Yin, X.; Scalia, A.; Samara, Y.N.; Stearns, R.; Vlahos, H.; Ellson, R.; Sweet, R.M.; Soares, A.S. Acoustic Methods to Monitor Protein Crystallization and to Detect Protein Crystals in Suspensions of Agarose and Lipidic Cubic Phase. J. Lab. Autom. 2016, 21, 107-114. [CrossRef] [PubMed] 
5. Luft, J.R.; DeTitta, G.T. Chaperone salts, polyethylene glycol and rates of equilibration in vapor-diffusion crystallization. Acta Crystallogr. Sect. D 1995, 51, 780-785. [CrossRef] [PubMed]

6. Stojanoff, V.; Jakoncic, J.; Oren, D.A.; Nagarajan, V.; Navarro Poulsen, J.C.; Adams-Cioaba, M.A.; Bergfors, T.; Sommer, M.O. From screen to structure with a harvestable microfluidic device. Acta Crystallogr. Sect. F 2011, 67, 971-975. [CrossRef] [PubMed]

7. McPherson, A.; Gavira, J.A. Introduction to protein crystallization. Acta Crystallogr. Sect. F 2014, 70, 2-20. [CrossRef] [PubMed]

8. Robert, M.C.; Lefaucheux, F. Crystal growth in gels: Principle and applications. J. Cryst. Growth 1988, 90, 358-367. [CrossRef]

9. Provost, K.; Robert, M.C. Application of gel growth to hanging drop technique. J. Cryst. Growth 1991, 110, 258-264. [CrossRef]

10. Snell, E.H.; Helliwell, J.R. Macromolecular crystallization in microgravity. Rep. Prog. Phys. 2005, 68, 799. [CrossRef]

11. McPherson, A. Review Current approaches to macromolecular crystallization. In EJB Reviews 1990; Springer: Berlin/Heidelberg, Germany, 1990; pp. 49-71.

12. Salemme, F.R. A free interface diffusion technique for the crystallization of proteins for X-ray crystallography. Arch. Biochem. Biophys. 1972, 151, 533-539. [CrossRef]

13. Gorrec, F.; Löwe, J. Automated Protocols for Macromolecular Crystallization at the MRC Laboratory of Molecular Biology. J. Vis. Exp. 2018. [CrossRef] [PubMed]

14. Hassell, A.M.; An, G.; Bledsoe, R.K.; Bynum, J.M.; Carter, H.L.; Deng, S.J.; Gampe, R.T.; Grisard, T.E.; Madauss, K.P.; Nolte, R.T.; et al. Crystallization of protein-ligand complexes. Acta Crystallogr. Sect. D Biol. Crystallogr. 2007, 63, 72-79. [CrossRef] [PubMed]

15. Cole, K.; Roessler, C.G.; Mule, E.A.; Benson-Xu, E.J.; Mullen, J.D.; Le, B.A.; Tieman, A.M.; Birone, C.; Brown, M.; Hernandez, J.; et al. A linear relationship between crystal size and fragment binding time observed crystallographically: Implications for fragment library screening using acoustic droplet ejection. PLoS ONE 2014, 9, e101036. [CrossRef] [PubMed]

16. Ehrmann, F.R.; Stojko, J.; Metz, A.; Debaene, F.; Barandun, L.J.; Heine, A.; Diederich, F.; Cianférani, S.; Reuter, K.; Klebe, G. Soaking suggests "alternative facts": Only co-crystallization discloses major ligandinduced interface rearrangements of a homodimeric tRNA-binding protein indicating a novel mode-ofinhibition. PLoS ONE 2017, 12, e0175723. [CrossRef] [PubMed]

17. Moreno, A.; Quiroz-García, B.; Yokaichiya, F.; Stojanoff, V.; Rudolph, P. Protein crystal growth in gels and stationary magnetic fields. Cryst. Res. Technol. J. Exp. Ind. Crystallogr. 2007, 42, 231-236. [CrossRef]

18. Thomas, D.H.; Rob, A.; Rice, D.W. A novel dialysis procedure for the crystallization of proteins. Protein Eng. Des. Sel. 1989, 2, 489-491. [CrossRef]

19. Pippard, M.J. Iron deficiency anemia, anemia of chronic disorders and iron overload. In Blood and Bone Marrow Pathology, 2nd ed.; Elsevier: Amsterdam, The Netherlands, 2011; pp. 173-195.

20. Honarmand Ebrahimi, K.; Hagedoorn, P.L.; Hagen, W.R. Unity in the biochemistry of the iron-storage proteins ferritin and bacterioferritin. Chem. Rev. 2014, 115, 295-326. [CrossRef] [PubMed]

21. Arosio, P.; Elia, L.; Poli, M. Ferritin, cellular iron storage and regulation. IUBMB Life 2017, 69, 414-422. [CrossRef]

22. Weiss, G. Iron and immunity: A double-edged sword. Eur. J. Clin. Investig. 2002, 32, 70-78. [CrossRef]

23. Arosio, P.; Levi, S. Ferritin, iron homeostasis, and oxidative damage 1, 2. Free Radic. Biol. Med. 2002, 33, 457-463. [CrossRef]

24. Jutz, G.; van Rijn, P.; Santos Miranda, B.; Böker, A. Ferritin: A versatile building block for bionanotechnology. Chem. Rev. 2015, 115, 1653-1701. [CrossRef] [PubMed]

25. Zipper, L.E.; Aristide, X.; Bishop, D.P.; Joshi, I.; Kharzeev, J.; Patel, K.B.; Santiago, B.M.; Joshi, K.; Dorsinvil, K.; Sweet, R.M.; et al. A simple technique to reduce evaporation of crystallization droplets by using plate lids with apertures for adding liquids. Acta Crystallogr. Sect. F 2014, 70, 1707-1713. [CrossRef] [PubMed]

26. Teplitsky, E.; Joshi, K.; Ericson, D.L.; Scalia, A.; Mullen, J.D.; Sweet, R.M.; Soares, A.S. High throughput screening using acoustic droplet ejection to combine protein crystals and chemical libraries on crystallization plates at high density. J. Struct. Biol. 2015, 191, 49-58. [CrossRef] [PubMed] 
27. Gil-Alvaradejo, G.; Ruiz-Arellano, R.R.; Owen, C.; Rodríguez-Romero, A.; Rudino-Pinera, E.; Antwi, M.K.; Stojanoff, V.; Moreno, A. Novel protein crystal growth electrochemical cell for applications in X-ray diffraction and atomic force microscopy. Cryst. Growth Des. 2011, 11, 3917-3922. [CrossRef]

28. Kabsch, W. Xds. Acta Crystallogr. Sect. D Biol. Crystallogr. 2010, 66, 125-132. [CrossRef] [PubMed]

29. Winn, M.D.; Ballard, C.C.; Cowtan, K.D.; Dodson, E.J.; Emsley, P.; Evans, P.R.; Keegan, R.M.; Krissinel, E.B.; Leslie, A.G.W.; McCoy, A.; et al. Overview of the CCP4 suite and current developments. Acta Crystallogr. Sect. D 2011, 67, 235-242. [CrossRef]

30. Winn, M.D.; Murshudov, G.N.; Papiz, M.Z. Macromolecular TLS refinement in REFMAC at moderate resolutions. Methods Enzymol. 2003, 374, 300-321.

31. Lamzin, V.S.; Wilson, K.S. Automated refinement of protein models. Acta Crystallogr. Sect. D Biol. Crystallogr. 1993, 49, 129-147. [CrossRef]

32. Granier, T.; Gallois, B.; Dautant, A.; Langlois d'Estaintot, B.; Precigoux, G. Comparison of the Structures of the Cubic and Tetragonal Forms of Horse-Spleen Apoferritin. Acta Crystallogr. Sect. D 1997, 53, $580-587$. [CrossRef]

33. Emsley, P.; Cowtan, K. Coot: Model-building tools for molecular graphics. Acta Crystallogr. Sect. D Biol. Crystallogr. 2004, 60, 2126-2132. [CrossRef]

34. Yin, X.; Scalia, A.; Leroy, L.; Cuttitta, C.M.; Polizzo, G.M.; Ericson, D.L.; Roessler, C.G.; Campos, O.; Ma, M.Y.; Agarwal, R.; et al. Hitting the target: Fragment screening with acoustic in situ co-crystallization of proteins plus fragment libraries on pin-mounted data-collection micromeshes. Acta Crystallogr. Sect. D 2014, 70, 1177-1189. [CrossRef] [PubMed]

35. Folzer, E.; Diepold, K.; Bomans, K.; Finkler, C.; Schmidt, R.; Bulau, P.; Huwyler, J.; Mahler, H.C.; Koulov, A.V. Selective Oxidation of Methionine and Tryptophan Residues in a Therapeutic IgG1 Molecule. J. Pharm. Sci. 2015, 104, 2824-2831. [CrossRef] [PubMed]

36. Soares, A.S.; Caspar, D.L. X-ray diffraction measurement of cosolvent accessible volume in rhombohedral insulin crystals. J. Struct. Biol. 2017, 200, 213-218. [CrossRef] [PubMed]

37. Bolann, B.J.; Ulvik, R.J. On the limited ability of superoxide to release iron from ferritin. Eur. J. Biochem. 1990, 193, 899-904. [CrossRef] [PubMed]

38. Smith, J.M.; Helliwell, J.R.; Papiz, M.Z. Absorption of X-radiation by single crystals of proteins containing labile metal components: The determination of the number of iron atoms within the central core of ferritin. Inorg. Chim. Acta 1985, 106, 193-196. [CrossRef] 